\title{
Characterisation and classification of oligometastatic disease: a European Society for Radiotherapy and Oncology and European Organisation for Research and Treatment of Cancer consensus recommendation
}

Citation for published version (APA):

Guckenberger, M., Lievens, Y., Bouma, A. B., Collette, L., Dekker, A., deSouza, N. M., Dingemans, A. M. C., Fournier, B., Hurkmans, C., Lecouvet, F. E., Meattini, I., Romero, A. M., Ricardi, U., Russell, N. S., Schanne, D. H., Scorsetti, M., Tombal, B., Verellen, D., Verfaillie, C., \& Ost, P. (2020). Characterisation and classification of oligometastatic disease: a European Society for Radiotherapy and Oncology and European Organisation for Research and Treatment of Cancer consensus recommendation. Lancet oncology, 21(1), E18-E28. https://doi.org/10.1016/S1470-2045(19)30718-1

Document status and date:

Published: 01/01/2020

DOI:

10.1016/S1470-2045(19)30718-1

Document Version:

Publisher's PDF, also known as Version of record

Document license:

Taverne

Please check the document version of this publication:

- A submitted manuscript is the version of the article upon submission and before peer-review. There can be important differences between the submitted version and the official published version of record. People interested in the research are advised to contact the author for the final version of the publication, or visit the DOI to the publisher's website.

- The final author version and the galley proof are versions of the publication after peer review.

- The final published version features the final layout of the paper including the volume, issue and page numbers.

Link to publication

\footnotetext{
General rights rights.

- You may freely distribute the URL identifying the publication in the public portal. please follow below link for the End User Agreement:

www.umlib.nl/taverne-license

Take down policy

If you believe that this document breaches copyright please contact us at:

repository@maastrichtuniversity.nl

providing details and we will investigate your claim.
}

Copyright and moral rights for the publications made accessible in the public portal are retained by the authors and/or other copyright owners and it is a condition of accessing publications that users recognise and abide by the legal requirements associated with these

- Users may download and print one copy of any publication from the public portal for the purpose of private study or research.

- You may not further distribute the material or use it for any profit-making activity or commercial gain

If the publication is distributed under the terms of Article 25fa of the Dutch Copyright Act, indicated by the "Taverne" license above,

Download date: 26 Apr. 2023 


\title{
Characterisation and classification of oligometastatic disease: a European Society for Radiotherapy and Oncology and European Organisation for Research and Treatment of Cancer consensus recommendation
}

\author{
Matthias Guckenberger, Yolande Lievens, Angelique B Bouma, Laurence Collette, Andre Dekker, Nandita M deSouza, Anne-Marie C Dingemans, \\ Beatrice Fournier, Coen Hurkmans, Frédéric E Lecouvet, Icro Meattini, Alejandra Méndez Romero, Umberto Ricardi, Nicola S Russell, \\ Daniel H Schanne, Marta Scorsetti, Bertrand Tombal, Dirk Verellen, Christine Verfaillie, Piet Ost
}

\begin{abstract}
Oligometastatic disease has been proposed as an intermediate state between localised and systemically metastasised disease. In the absence of randomised phase 3 trials, early clinical studies show improved survival when radical local therapy is added to standard systemic therapy for oligometastatic disease. However, since no biomarker for the identification of patients with true oligometastatic disease is clinically available, the diagnosis of oligometastatic disease is based solely on imaging findings. A small number of metastases on imaging could represent different clinical scenarios, which are associated with different prognoses and might require different treatment strategies. 20 international experts including 19 members of the European Society for Radiotherapy and Oncology and European Organisation for Research and Treatment of Cancer OligoCare project developed a comprehensive system for characterisation and classification of oligometastatic disease. We first did a systematic review of the literature to identify inclusion and exclusion criteria of prospective interventional oligometastatic disease clinical trials. Next, we used a Delphi consensus process to select a total of 17 oligometastatic disease characterisation factors that should be assessed in all patients treated with radical local therapy for oligometastatic disease, both within and outside of clinical trials. Using a second round of the Delphi method, we established a decision tree for oligometastatic disease classification together with a nomenclature. We agreed oligometastatic disease as the overall umbrella term. A history of polymetastatic disease before diagnosis of oligometastatic disease was used as the criterion to differentiate between induced oligometastatic disease (previous history of polymetastatic disease) and genuine oligometastatic disease (no history of polymetastatic disease). We further subclassified genuine oligometastatic disease into repeat oligometastatic disease (previous history of oligometastatic disease) and de-novo oligometastatic disease (first time diagnosis of oligometastatic disease). In de-novo oligometastatic disease, we differentiated between synchronous and metachronous oligometastatic disease. We did a final subclassification into oligorecurrence, oligoprogression, and oligopersistence, considering whether oligometastatic disease is diagnosed during a treatment-free interval or during active systemic therapy and whether or not an oligometastatic lesion is progressing on current imaging. This oligometastatic disease classification and nomenclature needs to be prospectively evaluated by the OligoCare study.
\end{abstract}

\section{Introduction}

Hellman and Weichselbaum ${ }^{1}$ first proposed oligometastatic disease as a distinct cancer state between locally confined and systemically metastasised disease in 1995. In such patients with limited metastatic disease, the value of integrating local metastases-directed therapy into the treatment framework has been investigated in five randomised phase 2 studies. Three of these studies ${ }^{2-4}$ have assessed whether or not the addition of metastasesdirected local therapy to standard-of-care systemic therapy improves outcome in oligometastatic disease, as compared with systemic treatment alone. All three studies reported improved progression-free survival ${ }^{2}$ or overall survival ${ }^{3,4}$ with the addition of metastases-directed local therapy. Palma and colleagues ${ }^{5}$ described an overall survival benefit of metastases-directed stereotactic body radiotherapy in addition to standard of care for patients with oligometastatic disease with controlled primary malignancy in a tumour-agnostic trial that mostly involved patients with breast, lung, colorectal, and prostate cancer. Ost and colleagues ${ }^{6}$ did a study in patients with oligorecurrent prostate cancer, in which metastases-directed stereotactic body radiotherapy was compared with surveillance; systemic therapy in the form of androgen deprivation was not a component of the initial treatment strategy but was used only at disease progression. Androgen deprivation therapy-free survival was longer with metastasis-directed therapy than with surveillance alone. In another prostate cancer study, the randomised phase 3 STAMPEDE trial, the investigators addressed the hypothesis that local treatment of the primary tumour alone, without metastases-directed therapy, affects outcome in metastatic disease. Local radiotherapy of the prostate was shown to improve overall survival in patients with a low metastatic burden, but not in those with a high metastatic burden, compared with androgen deprivation therapy only.

Conversely, less progress has been made in understanding and defining oligometastatic disease based on tumour biology (ie, in recognising patients with truly limited metastatic capacity by oligometastatic disease-specific biomarkers). ${ }^{8}$ MicroRNA profiles, which
Lancet Oncol 2020; 21: e18-28 Department of Radiation Oncology, University Hospital Zurich, University of Zurich, Zurich, Switzerland (Prof M Guckenberger MD, D H Schanne MD); Department for Radiation Oncology, Ghen University Hospital and Ghent University, Ghent, Belgium (ProfY Lievens PhD Prof P Ost PhD); European Organisation for Research and Treatment of Cancer Headquarters, Brussels, Belgium (A B Bouma MD, L Collette PhD, B Fournier PhD): Department of Radiation Oncology, School for Oncology and Developmental Biology (A Dekker PhD), and Department of Respiratory Medicine

(Prof A-M C Dingemans PhD), Maastricht University Medical Centre, Maastricht, Netherlands; Division of Radiotherapy and Imaging, Institute of Cancer Research, Royal Marsden Hospital, London, UK (Prof N M deSouza FRCR). Department of Respiratory Medicine, Erasmus Medical Center, Rotterdam, Netherlands (Prof A-M C Dingemans); Department of Radiotherapy, Catharina Ziekenhuis, Eindhoven, Netherlands (C Hurkmans PhD); Radiology Department, Institut de Recherche Expérimentale et Clinique Cliniques Universitaires Saint Luc, Université Catholique de Louvain, Brussels, Belgium (Prof F E Lecouvet PhD); Department of Experimental and Clinical Biomedical Sciences, University of Florence, Florence, Italy (Prof I Meattini MD); Radiation Oncology Unit, Azienda Ospedaliero-Universitaria Careggi, Florence, Italy 
(Prof I Meattini); Department of Radiation Oncology, Erasmus Medical Center, University Medical Center, Rotterdam, Netherlands

(A Mendez Romero PhD); Department of Oncology, University of Turin, Turin, Italy (Prof U Ricardi MD); Division of Radiotherapy, Netherlands Cancer Institute, Amsterdam, Netherlands (N S Russell PhD); Radiotherapy and Radiosurgery Department, Humanitas Clinical and Research Hospital, Istituto di Ricovero e Cura a Carattere Scientifico, Milan, Italy (Prof M Scorsetti MD); Department of Urology, Cliniques Universitaires Saint Luc, Université Catholique de Louvain, Louvain-la-Neuve, Brussels, Belgium (Prof B Tombal PhD); Iridium Kankernetwerk and University of Antwerp, Faculty of Medicine and Health

Sciences, Antwerp, Belgium (Prof D Verellen PhD); and European Society for

Radiotherapy and Oncology Headquarters, Brussels, Belgium (CVerfaillie) Correspondence to:

Prof Matthias Guckenberger, Department of Radiation Oncology, University Hospital Zurich, University of Zurich, CH - 8091 Zurich, Switzerland matthias.guckenberger@usz. correlate with progression or differentiate oligometastatic and polymetastatic lung disease, have been reported ${ }^{9,10}$ as has an integrated molecular subtype for identifying a curable oligometastatic state in colorectal liver metastasis. ${ }^{11}$ Dhondt and colleagues ${ }^{12}$ described a microRNA signature to identify oligometastatic prostate cancer. However, external or independent validation of these biomarkers has been either unsuccessful or is still absent.

The current scarcity of biomarkers has made imaging the most relevant diagnostic method for defining oligometastatic disease. ${ }^{13}$ Rapid advances in imaging that allow identification of small metastases help to differentiate between oligometastatic and polymetastatic disease, thereby excluding patients with more widespread disease from unnecessary local treatment. For example, fluorodeoxyglucose PET has been shown to improve the selection of patients with a low tumour burden in nonsmall-cell lung cancer and colorectal cancer ${ }^{14,15}$ who might benefit the most from radical local metastases-directed treatment. The European Organisation for Research and Treatment of Cancer (EORTC) has identified the crucial role of imaging to standardise and optimise the clinical diagnosis of oligometastatic disease and has published expert recommendations. ${ }^{16,17}$ Although these recommendations are intended to assist in the design of clinical trials, European Society for Medical Oncology (ESMO) clinical practice guidelines already refer to these imaging recommendations. ${ }^{18}$ Uncertainty remains with regards to the exact meaning of limited metastases; however, trial design and clinical practice today are rather consistent in limiting oligometastatic disease to a maximum of three to five metastases. ${ }^{19}$ Despite these improvements in imaging and some consensus on imaging-based definition of oligometastatic disease, clinical outcome after treatment of oligometastatic disease varies substantially: a systematic literature review in oligometastatic non-small-cell lung cancer reported that 5-year overall survival ranges between $86 \%$ and $8 \cdot 3 \% .^{20}$ This range is equivalent to the variation in overall survival between stage I and stage IV non-small-cell lung cancer. ${ }^{21}$ Several studies have proposed prognostic scoring systems or nomograms to achieve more accurate overall survival prediction and patient selection; ${ }^{22-25}$ however, all these studies are based on retrospective cohorts with potential bias in patient selection and none have been prospectively validated.

The limitations of imaging, or more precisely its clinical interpretation, could substantially affect clinical outcomes. In fact, Hellman and Weichselbaum described in their landmark paper two very different clinical scenarios, both under the umbrella of oligometastases: "tumors early in the chain of progression with metastases limited in number and location" and "another group of patients with oligometastases who had widespread metastases that were mostly eradicated by systemic agents, the chemotherapy having failed to destroy those remaining because of the number of tumour cells, the presence of drug-resistant cells, or the tumour foci being located in some pharmacologically privileged site". These two scenarios of oligometastatic disease, which might present with similar features on imaging but differ substantially from a clinical perspective, are most likely associated with very different outcome and require different treatment strategies. Consequently, better characterisation and classification of the different states of oligometastatic disease is needed.

We have convened a group of international experts in diagnosis and treatment of oligometastatic disease from the EORTC and European Society for Radiotherapy and Oncology (ESTRO) OligoCare project and initiated this consensus process on characterisation and classification of oligometastatic disease. We aimed to establish a comprehensive system of oligometastatic disease characterisation factors, which should be assessed in all patients treated with radical local treatment for oligometastatic disease both inside and outside of clinical trials. These oligometastatic disease characterisation factors were the basis to develop and agree on an oligometastatic disease classification system and nomenclature that cover all possible clinical situations of imaging findings with few metastases. The classification system should be unambiguous, based on established prognostic patient and disease characteristics, and not require additional diagnostic testing. The classification system should also reflect fundamental biological and clinical processes underlying the development of oligometastatic disease and should be independent from the primary tumour type.

\section{Methods}

\section{Data collection}

This project originates from the ESTRO and EORTC OligoCare registry project (EORTC 1822, first cohort of the joint EORTC-ESTRO RADiation InfrAstrucTure for Europe-E2-RADIatE, EORTC 1811, NCT03818503) which aims to identify patient, tumour, staging, and treatment characteristics that affect overall survival of patients treated with metastases-directed radiotherapy for oligometastatic disease. The inclusion criteria for this project are broad to represent the diversity of daily clinical practice and to allow the identification of relevant prognostic and predictive factors. Patients are eligible irrespective of whether oligometastatic disease is diagnosed synchronously or metachronously and regardless of previous surgical, locally ablative therapy, and systemic treatments. Included patients can have been treated previously for oligometastatic or nonoligometastatic disease.

We defined factors for oligometastatic disease characterisation in a two-step process, starting with a systematic literature review followed by a Delphi consensus process. All coauthors of this manuscript 
(except DHS) are members of the OligoCare project and represent ESTRO or EORTC as experts in clinical trial design, diagnosis, and treatment of oligometastatic disease. All 19 members of the OligoCare project contributed to all parts of the Delphi consensus definition process.

\section{Search strategy and selection criteria}

We followed PRISMA guidelines for this systematic literature review. ${ }^{26}$ PubMed and Embase were searched for prospective clinical trials on oligometastatic disease published in English. Two investigators (MG and DHS) independently searched the databases up to March 24, 2019. The search terms were: (["oligometastasis" OR “oligometastatic" OR "oligometastases" OR "oligorecurrence" OR "oligorecurrent" OR "oligoprogression" OR “oligoprogressive” OR "oligopersistent” OR “oligopersistence"] AND ["randomised" OR "randomized" OR "prospective"]). We also reviewed the references of articles included in the final selection. To be eligible, trials had to be prospective phase 1-3 studies (to reduce bias), be therapeutic interventional studies, and report outcome of overall survival or progression-free survival, or disease recurrence. Studies reporting quality of life only or studies limited to brain metastases were excluded. Two investigators (MG and DHS) independently reviewed the list of retrieved articles and selected potentially relevant articles. The same two investigators independently extracted study inclusion and exclusion criteria data from all selected studies. We did not extract general patient characteristics unrelated to oligometastatic disease (eg, age, sex, or performance status) or comorbidity data. We considered these study inclusion and exclusion criteria as factors for oligometastatic disease characterisation and included them into the Delphi process for consensus definition.

\section{Consensus formation}

We used a Delphi process to establish consensus about oligometastatic disease characterisation factors. ${ }^{27}$ We circulated surveys to all individual participants using the online survey tools SurveyMonkey and Google Forms. In round one, the participants were provided with the results of the systematic review. The participants were asked to answer one open-ended question: to describe potential oligometastatic disease characterisation factors and classification factors that had not been identified in the systematic review. We assumed that simultaneous or secondary primaries or non-malignant lesions had been excluded by clinical judgment, repeated or multi-method imaging, or biopsy confirmation. Two investigators (MG and DHS) consolidated all the participants' responses into a list of oligometastatic disease characteristics that was circulated to all participants in the second round of the Delphi process to assess whether or not consolidation did introduce misinterpretations of individual responses. In the third round, all oligometastatic disease characterisation factors, extracted from the systematic review and from the first two Delphi rounds, were provided to the participants to score each item on a 5-point Likert scale (1=strongly agree; $5=$ strongly disagree); for each item, we asked: "should the oligometastatic disease characterisation factor be assessed in all patients treated with radical local treatment for oligometastatic disease inside and outside of clinical trials and form the basis for the case report forms of the prospective OligoCare registry trial, assessing real-world practice and outcome in this setting?”. A threshold of $75 \%$ or more for agreement or disagreement was required for each item to reach consensus. We analysed all responses and comments, recorded items reaching consensus and did not include these in the subsequent survey. Next, we asked the participants to vote again on items that had not reached $75 \%$ or more agreement. Following this round, we excluded from the final recommendations any item that still did not have consensus. We generated a consensus of oligometastatic disease characterisation factors by combining all items that reached consensus. During this phase, only minor modifications to grammar and wording were accepted. No additions or removals of items were permitted.

We developed the oligometastatic disease classification system in a three-step process. First, descriptive tumour and treatment characteristics, quantitative oligometastatic disease characteristics, and characteristics relating to individual metastases were excluded as potential oligometastatic disease classification factors. The remaining oligometastatic disease characteristics were all addressing the process of oligometastatic disease development and were formulated as binary yes or no questions. Second, a decision tree was established on the basis of binary questions. The decision tree started with imaging-based diagnosis of oligometastatic disease and the hierarchical order aimed to minimise the number of branches and considered the temporal course of oligometastatic disease development. Lastly, nomenclatures of previously proposed oligometastatic states ${ }^{1,28-33}$ were applied to the decision tree and complemented two of the oligometastatic disease states, which remained unaddressed in the literature.

We assessed the oligometastatic disease classification system for consensus in a Delphi process: we provided the participants with the graphical overview of the classification system in form of a decision tree and each participant scored each oligometastatic disease state on a five-point Likert scale (1=strongly agree; 5=strongly disagree). We set a threshold of $75 \%$ or more for agreement or disagreement for each item to reach consensus. In a second round, we provided the participants with the anonymous responses from the previous round. Following this round, any item still without consensus was excluded from final recommendations. 


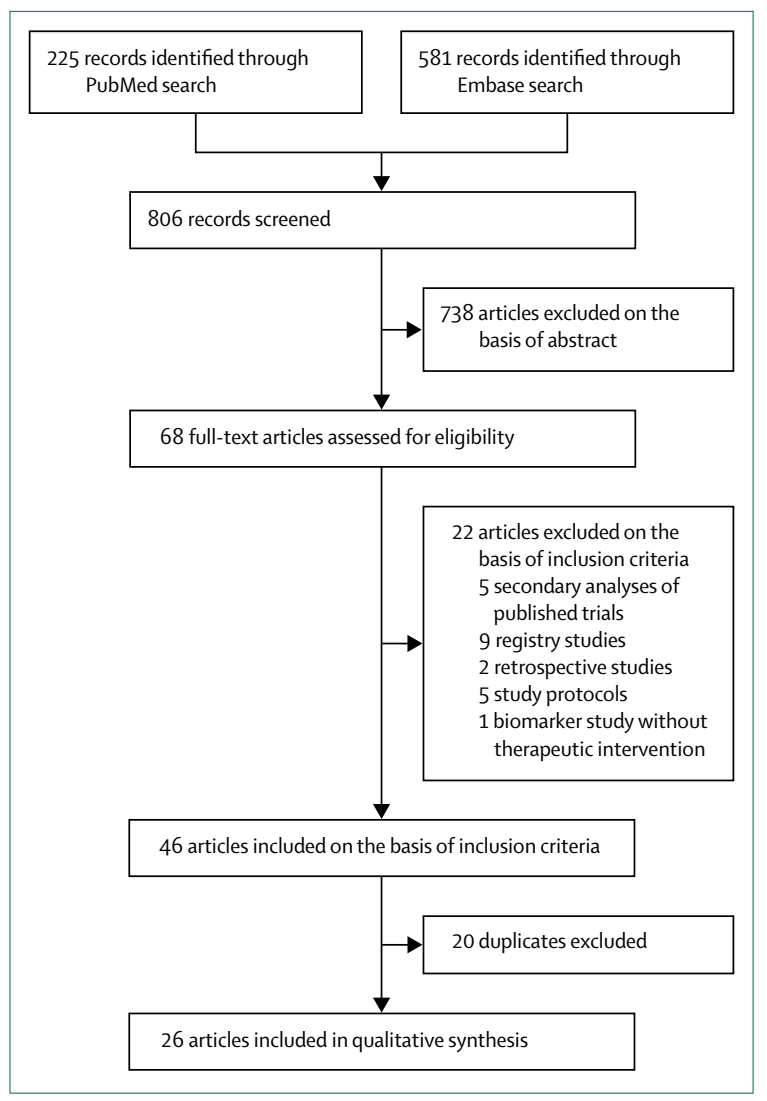

Figure 1: Flowchart of article selection process

\section{Findings}

\section{Oligometastatic disease characterisation}

The systematic review retrieved 806 publications, from which we selected 68 potentially relevant articles after abstract screening. After full-text article review, 46 manuscripts fulfilled the inclusion criteria, and after exclusion of 20 duplicate records or repeated publications of identical clinical trials, 26 studies reporting overall survival or progression-free survival of prospective interventional trials for oligometastatic disease were analysed (figure 1). We identified a total of ten oligometastatic disease-related study inclusion and exclusion criteria in these 26 prospective trials, which were potential candidates for oligometastatic disease charSee Online for appendix acterisation factors (appendix pp 2-3).

In the first two rounds of the Delphi process (figure 2, appendix pp 4-5), we collected eight additional candidates for oligometastatic disease characterisation and combined invasive staging with imaging-based staging arriving at a total of 18 characteristics, which were scored independently in rounds $3 \cdot 1$ and 3.2 of the Delphi process (figure 2, appendix pp 6-11). After this Delphi consensus process, we agreed upon all ten oligometastatic disease characteristics identified in the systematic review and added seven additional characteristics to the final list of 17 oligometastatic disease characteristics (panel). The
Step 1

April 1, 2019, 100\% response rate

All 19 participants were asked to answer one open question: to describe potential oligometastasic disease characterisation factors and classification factors in addition to the ones identified in the systematic review

\begin{tabular}{|l|}
\hline $\begin{array}{l}\text { Step } 2 \\
\text { April-May, 2019, 100\% response rate } \\
\text { The consolidated list of nine additional oligometastasis disease characteristics } \\
\text { was circulated to all participants who could then agree or disagree on } \\
\text { consolidation; consolidation was approved for all items }\end{array}$ \\
$\begin{array}{l}\text { Step 3.1 } \\
\text { May-June, 2019, 89\% response rate } \\
\text { All ten oligometastasis disease characterisation factors from the systematic } \\
\text { review and eight from the first two Delphi rounds were provided to the } \\
\text { participants to score each item on a 5-point Likert scale; 17 items were } \\
\text { approved }\end{array}$ \\
$\begin{array}{l}\text { Step 3.2 } \\
\text { June, 2019, 100\% response rate } \\
\text { Participants were asked to vote again on one single item ("Is the } \\
\text { oligometastasic lesion pathologically proven?") that had not reached } \\
>75 \% \text { agreement; this item was not approved }\end{array}$ \\
\hline $\begin{array}{l}\text { Step } 4 \\
\text { June-July, 2019, 95\% response rate } \\
\text { disease classification system in the form of a decision tree and each } \\
\text { oligometastasis disease state was scored by each participant on a 5-point } \\
\text { Likert scale; all items were approved }\end{array}$ \\
\hline
\end{tabular}

Figure 2: Schematic overview of the Delphi process

response rate of the 19 experts during the Delphi process was at least $89 \%$ for all rounds of the process (figure 2).

Oligometastatic disease characterisation classification

We established and agreed the oligometastatic disease classification system on the basis of the previously agreed characterisation system. After discussion, 17 (94\%) of 18 participants agreed (either strongly agreed or agreed) on oligometastatic disease as the umbrella term for all states of limited metastatic disease, staying within the tradition of the original publication of Hellman and Weichselbaum. ${ }^{1}$ Differentiation is based on five characterisation factors which we identified as the basis for the oligometastatic disease classification system and decision tree comprising of a total of eight branches and a total of nine distinct states of oligometastatic disease (figure 3 and figure 4).

\section{Question 1: Does the patient have a history of polymetastatic disease before current diagnosis of oligometastatic disease?}

This question differentiates between genuine oligometastatic disease (patients without a history of polymetastatic disease) and induced oligometastatic disease (patients with a history of polymetastatic disease). In genuine oligometastatic disease, the absence of polymetastatic disease in the patient's history indicates a 
low metastatic capacity of cancer. Induced oligometastatic disease has already been described by Hellman and Weichselbaum:1 "the chemotherapy (or more in general systemic therapy) having failed to destroy (or control) those remaining (metastases) because of the number of tumour cells, the presence of drug-resistant cells, or the tumour foci being located in some pharmacologically privileged site". Induced oligometastatic disease does therefore not indicate a possible low metastatic capacity, as is the case in genuine oligometastatic disease, but rather a state of disease, which is the result of polymetastatic disease treated with systemic therapy with or without local therapy.

\section{Question 2: Does the patient have a history of oligometastatic disease before the current diagnosis of oligometastatic disease?}

For patients with genuine oligometastatic disease, this question differentiates between de-novo oligometastatic disease (patients without a previous diagnosis of oligometastatic disease) and repeat oligometastatic disease (patients with a previous diagnosis of oligometastatic disease). De-novo oligometastatic disease is the classic state of the disease as initially described by Hellman and Weichselbaum. ${ }^{1}$ For patients with a previous diagnosis of oligometastatic disease, who have not progressed to polymetastatic disease after failure to local and systemic treatment, repeat oligometastatic disease might represent a cancer with favourable biology of low metastatic capacity over a long period of time. Polymetastatic disease is the most frequent failure pattern after treatment of oligometastatic disease, but some studies report that repeat oligometastatic disease is observed in $27-75 \%$. $^{3436}$

\section{Question 3: Has oligometastatic disease been first diagnosed more than 6 months after the primary cancer diagnosis?}

For patients with de-novo oligometastatic disease, this question differentiates between synchronous oligometastatic disease (maximum 6 months interval between diagnosis of oligometastatic disease and primary cancer diagnosis) and metachronous oligometastatic disease (more than 6 months interval between diagnosis of oligometastatic disease and primary cancer diagnosis). No consensus exists in the literature about the interval between primary cancer diagnosis and the development of oligometastatic disease to differentiate between synchronous and metachronous disease, ${ }^{29}$ however, diagnosis of oligometastatic disease more than 6 months after diagnosis of the primary tumour has been a frequently used definition for metachronous instead of synchronous oligometastatic diease. ${ }^{37,38}$ Although most studies report that synchronous oligometastatic disease is associated with a more aggressive disease phenotype and a worse prognosis than metachronous oligometastatic disease, ${ }^{22,39,40}$ these predictions were not confirmed by all studies. . $^{41,42}$
Panel: Characteristics of oligometastatic disease

\section{Descriptive tumour characteristics}

- Primary tumour characteristics: primary tumour site, histology, stage according to TNM Classification of Malignant Tumours, mutational status, tumour marker

- History of cancer progression: time interval since first diagnosis, disease-free interval, treatment-free interval

- History of treatment of primary tumour: method of local treatment, radical or palliative intent, controlled primary tumour

- History of systemic therapy before diagnosis of oligometastatic disease: types of systemic therapy, number of lines of systemic therapy

- Oligometastatic disease staging: imaging method, anatomical areas covered, invasive staging

- Involved organs of oligometastatic disease

\section{Quantitative characteristics}

- Number of metastatic lesions

- Number of involved organs

- Number of lesions per organ

- Maximum size or volume of individual metastasis

Developmental characteristics

- Does the patient have a history of polymetastatic disease before oligometastatic disease diagnosis?

- Does the patient have a history of oligometastatic disease before current diagnosis?

- Is oligometastatic disease diagnosed within 6 months after diagnosis of the primary tumour?

- Is the patient under active systemic therapy at the time of oligometastatic disease diagnosis?

- Are any oligometastatic lesions progressive on current imaging?

\section{Metastases-specific characteristics}

- Is the oligometastatic lesion a newly developed metastatic lesion?

- Is treatment of the oligometastatic lesion possible with radical intent?

Question 4: Is the patient under active systemic therapy at the time of oligometastatic disease diagnosis?

For patients with metachronous oligometastatic disease, repeat oligometastatic disease, and induced oligometastatic disease, development of oligometastatic disease in a treatment-free interval or during active systemic therapy is differentiated. Diagnosis of metachronous oligometastatic disease with the patient under active systemic therapy indicates metachronous oligoprogression. This state of oligometastatic disease is more frequently expected in those cancers that are treated with long-term systemic therapy at primary diagnosis (eg, androgen deprivation therapy for high-risk prostate cancer, endocrine therapy for breast cancer, or targeted therapy for driver-mutated non-small-cell lung cancer). The finding of repeat 


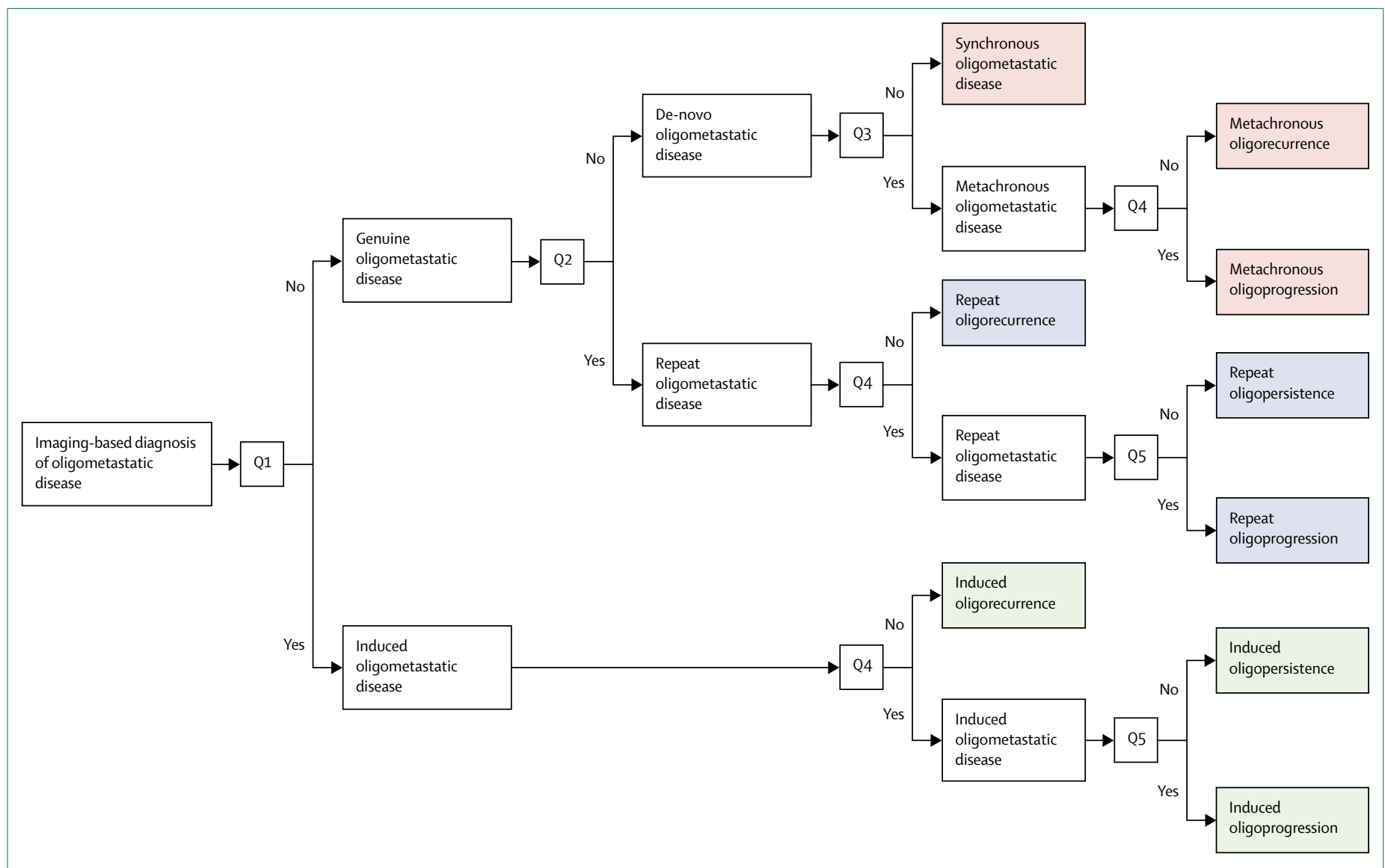

Figure 3: Decision tree for classification of oligometastatic disease

The decision tree starts with oligometastatic disease as umbrella term. Questions 1 and 2 differentiate between the upper-level oligometastatic states of de-novo (red), repeat (blue) and induced oligometastatic disease (green). Question 3 differentiates de-novo oligometastatic disease into synchronous and metachronous oligometastatic disease. Questions 4 and 5 subclassify into

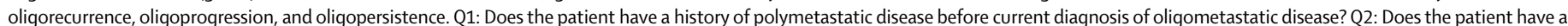

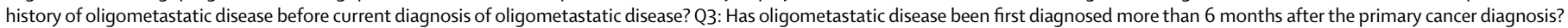
Q4: Is the patient under active systemic therapy at the time of oligometastatic disease diagnosis? Q5: Are any oligometastatic lesions progressive on current imaging?

oligometastatic disease and induced oligometastatic disease with the patient under active systemic therapy requires further sub-classification by question five and is explained below. Metachronous, repeat, and induced oligometastatic disease in patients not under active systemic therapy indicates oligorecurrence: the cancer responded well to local treatment, systemic treatment, or both, which allowed a treatment-free interval, and disease recurred later presenting as only a small number. The term oligo refers to the small number of growing or newly developed metastases; patients with induced oligorecurrence might have additional stable metastases, and the value of treating these stable metastases is currently unclear.

\section{Question 5: Are any oligometastatic lesions progressive on current imaging?}

For patients with repeat oligometastatic disease and induced oligometastatic disease under active systemic treatment, this question differentiates between oligoprogression (progressive disease on current imaging) and oligopersistence (stable disease or partial response on current imaging). The term oligo refers to a few growing or newly developed metastases (oligoprogression) or a few persistent metastases (oligopersistence). Patients with induced oligoprogression or induced oligopersistence might have additional metastases, which are controlled by or respond to systemic therapy; the value of treating these nonprogressive and nonpersistent metastases locally is currently unclear.

To the best of our knowledge, no data about the incidence and prognosis of repeat oligoprogression and repeat oligopersistence are available. Induced oligoprogression is a well-recognised state of oligometastatic disease: several studies have been done in patients with oligoprogressive non-small-cell lung cancer, in whom systemic therapy achieved good responses of polymetastatic disease but only a few metastases developed resistance and progressed later on; local ablative 
A De-novo oligometastatic disease Synchronous oligometastatic disease

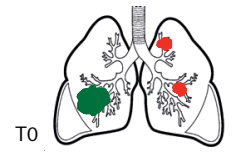

- T0: first time diagnosis of primary cancer (green) and oligometastases (red) within 6 months
B Repeat oligometastatic disease Repeat oligorecurrence

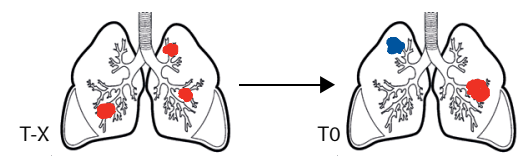

-T-X: diagnosis of oligometastases followed by local treatment or systemic treatment or both

- Systemic therapy-free interval

-T0: diagnosis of new (blue) and growing or regrowing (red) oligometastases

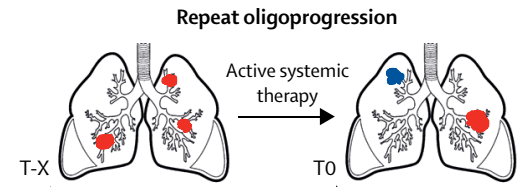

-T-X: diagnosis of oligometastases followed by local treatment or systemic treatment or both

- Under treatment with active systemic therapy

-T0: diagnosis of new (blue) and growing or regrowing (red)

oligometastases

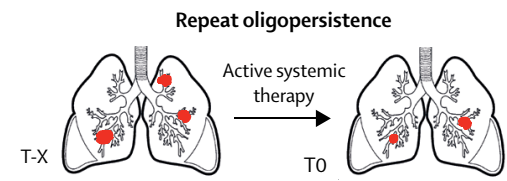

-T-X: diagnosis of oligometastases followed by local treatment or systemic treatment or both

- Under treatment with active systemic therapy

-T0: diagnosis of persistent non-progressive (red) oligometastases
C Induced oligometastatic disease Induced oligorecurrence

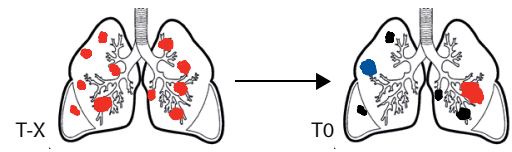

-T-X: diagnosis of polymetastatic metastatic disease followed by systemic treatment with or without local treatment - Systemic therapy-free interval

-T0: diagnosis of new (blue) and growing or regrowing (red) oligometastases, possible residual non-progressive metastases (black)

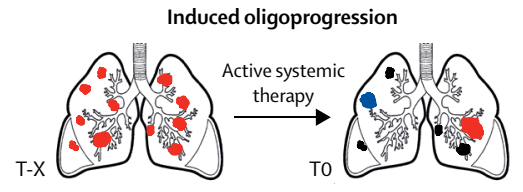

-T-X: diagnosis of polymetastatic metastatic disease followed by systemic treatment with or without local treatment - Under treatment with active systemic therapy -T0: diagnosis of new (blue) and growing or regrowing (red) oligometastases, possible residual non-progressive metastases (black)

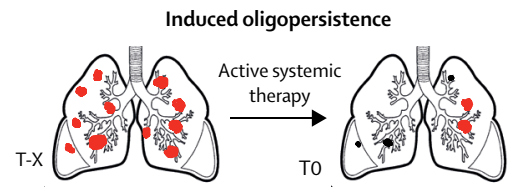

-T-X: diagnosis of polymetastatic metastatic disease followed by systemic treatment with or without local treatment - Under treatment with active systemic therapy

-TO: diagnosis of persistent non-progressive oligometastases (red), where response is worse compared with other residual metastases (black)

Figure 4: Illustration of the oligometastatic disease classification system

(A) De-novo oligometastatic disease. (B) Induced oligometastatic disease. (C) Repeat oligometastatic disease. In repeat and induced oligometastatic disease the primary tumour is assumed to be controlled by ongoing or previous treatment. Oligometastases are confirmed by imaging or biopsy to exclude simultaneous or secondary primary tumours. $\mathrm{T} 0=\mathrm{at}$ this current point of time. $\mathrm{T}-\mathrm{x}=\mathrm{any}$ previous point in time.

treatment was combined with either continuation of systemic therapy ${ }^{43-47}$ or switch to the next treatment line. ${ }^{48}$ In patients with induced oligopersistence, only stable disease or partial response is achieved in a few metastases, whereas a prolonged partial response or complete response is observed in the remaining polymetastatic disease. Both randomised trials in oligometastatic non-small-cell lung cancer enrolled patients with stage IV non-small-cell lung cancer after completing systemic therapy, irrespective of their tumour burden at their primary cancer diagnosis; the oligometastatic state was defined at the time of restaging after first-line therapy. ${ }^{2,4}$ Consequently, heterogeneous patients could have been recruited into these trials: ie, a mixture of patients with chemotherapy-resistant or targeted therapy-resistant genuine oligometastatic disease and patients with induced oligopersistence, in whom systemic treatment of polymetastatic disease achieved a complete response except for a few resistant metastases.

\section{Treatment strategies and goals}

Traditionally, local treatment for metastatic disease, irrespective of oligometastatic or polymetastatic state, was exclusively done with palliative intent. The systemic treatment strategy was dependent on several factors, including patient characteristics such as age and comorbidities; primary cancer type and molecular disease features; pattern, volume, and kinetics of disease progression; presence of symptoms; previous history of cancer treatment such as response to systemic treatment or disease-free interval; availability of current and future systemic therapy options, and their efficacy and toxicity profile; and patient's preference.

Both treatment goals and treatment strategies have changed with the introduction of the concept of oligometastatic disease. In clinical trials, overall survival, progression-free survival, avoidance of systemic therapy (eg, androgen-deprivation therapy in oligometastatic prostate cancer), and quality of life are the most frequently defined endpoints. ${ }^{31,49}$ Whereas local treatment 


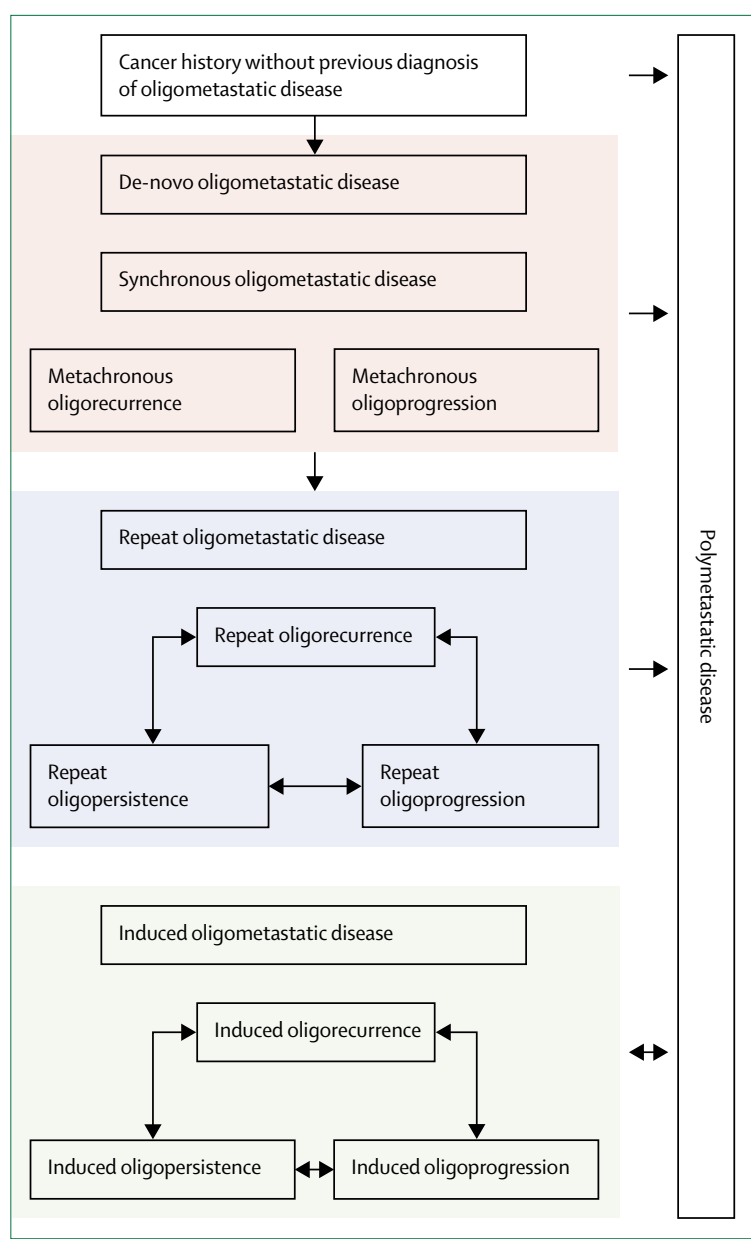

Figure 5: Dynamic oligometastatic state model

The model illustrates pathways for developing multiple and different states of oligometastatic disease throughout a patient's disease history. Patients might have de-novo oligometastatic disease or develop induced oligometastatic disease via polymetastatic disease as first-time diagnosis of oligometastatic disease. Repeat oligometastatic disease might develop as a degree of failure after treatment of de-novo oligometastatic disease; induced oligometastatic disease might develop after systemic failure following treatment of de-novo and repeat oligometastatic disease. Patients with repeat oligometastatic disease and induced oligometastatic disease might have dynamic transitions between oligorecurrent, oligoprogressive, and oligopersistent disease, depending on the response to local and systemic therapy.

aims to eradicate all oligometastases and the potentially uncontrolled primary tumour, the choice of the optimal combination strategy with systemic treatment depends on the oligometastatic disease state, the specific treatment goal, and the factors mentioned.

For all states of de-novo oligometastatic disease and repeat oligometastatic disease, radical treatment aims to achieve a status of freedom from disease. Translation of such status into prolonged overall survival or cure will depend on the efficacy of local treatment and on the absence or effective control of occult metastatic disease by the addition of systemic therapy. Consequently, all but one ${ }^{6}$ randomised trials in oligometastatic disease published so far used standard systemic therapy as a backbone of the oligometastatic disease treatment strategy. ${ }^{2-5}$ The choice of the optimal systemic therapy is especially unclear in metachronous oligoprogression, in which oligometastatic disease develops during active systemic therapy in the context of primary treatment, and continuation of systemic therapy or switching to another drug are sensible options. Synchronous oligometastatic disease adds another level of complexity because local treatment to the locoregional primary tumour, local treatment of all oligometastases, and systemic treatment all need to be combined into one treatment strategy. However, another goal of the local intervention in de-novo oligometastatic disease and repeat oligometastatic disease could be to prolong the time until systemic therapy is needed for polymetastatic disease and thereby maintain the patient's quality of life. This strategy has been tested for oligorecurrent prostate cancer, for which local metastases-directed therapy considerably prolonged the time until initiation of androgen deprivation therapy. ${ }^{6}$

Treatment goals and strategies are different in induced oligometastatic disease. These patients have polymetastatic disease, which is converted into a state of induced oligometastatic disease by partially effective systemic treatment. Local treatment for induced oligometastatic disease therefore complements the systemic treatment and not vice versa as is the case in genuine oligometastatic disease. Consequently, based on currently available evidence, cure is not achieved in most patients.

For patients with induced oligorecurrence, radical local treatment aims to restore a status of stable disease (in case of stable residual polymetastatic disease) or a status of complete response (in case of complete response of previous polymetastatic disease). The addition of systemic therapy could potentially enhance the effect of the local intervention. Whether systemic therapy is best performed as re-challenge with the previous line of treatment-which achieved stable disease or complete response and a systemic therapy-free interval thereafteror switch to the next line of treatment is unknown. Another goal of the local intervention could be to prolong the systemic therapy-free interval.

For patients with induced oligoprogression, radical local treatment aims to restore a status of overall sensitivity to systemic therapy through eradication of oligometastases with resistance to the current line of systemic therapy. For patients with induced oligopersistence, the goal of radical local treatment is to achieve an overall deeper response to systemic therapy by eradication of oligometastases with reduced sensitivity to the current line of systemic therapy. In both induced oligoprogression and induced oligopersistence, continuation of the current systemic therapy or switching to the next line of systemic therapy are possible options. The decision depends on the previous depth and duration of response, the volume and kinetics of progressive disease, associated symptoms, tolerability of the current and next 
line of systemic treatments, and likely efficacy of the next line of systemic treatment.

\section{Dynamic oligometastatic state model}

The proposed oligometastatic disease classification system defines the oligometastatic state at one timepoint in the patient's history. However, one patient might develop several and different states of oligometastatic disease throughout the course of disease, resulting in multiple courses of radical local and systemic treatment. Similar to the clinical states model proposed for prostate cancer, ${ }^{50}$ we therefore propose a dynamic oligometastatic state model (figure 5). First-time diagnosis of oligometastatic disease might occur as de-novo oligometastatic disease or as induced oligometastatic disease after systemic therapy of polymetastatic disease. Repeat oligometastatic disease might be the consequence of failure after treatment of de-novo oligometastatic disease, with failure being again limited to a few metastases. Following local ablative therapy with or without systemic therapy of de-novo or repeat oligometastatic disease, cancer might progress into polymetastatic disease. This situation will trigger initiation of systemic therapy, which might result in induced oligometastatic disease. Within repeat oligometastatic disease and within induced oligometastatic disease, patients can have dynamic transitions between oligorecurrent, oligoprogressive, and oligopersistent disease, depending on the response to local and systemic therapy.

For the three upper-level states of de-novo oligometastatic disease, repeat oligometastatic disease and induced oligometastatic disease, transition to any downstream state is possible, which is then unidirectional in the patient's history. Notwithstanding, transition from one oligometastatic state to another is not necessarily associated with a worsening of the prognosis. A patient with several courses of treatment for repeat oligometastatic disease most likely has a disease phenotype with truly limited metastatic capacity because no progression to polymetastases has developed. ${ }^{51}$ Additionally, patients with induced oligometastatic disease might have long-term survival when radical local treatment is combined with effective systemic treatment, such as targeting drugs for non-small-cell lung cancer with driver mutations or immunotherapy for malignant melanoma.

Clinical data support this dynamic oligometastatic state model with several sequential courses of radical local therapy for oligometastatic disease. A registry study on oligometastatic non-small-cell lung cancer reported that $6.6 \%$ of all patients treated for oligometastatic nonsmall-cell lung cancer received more than one course of treatment and a maximum of four courses of metastasesdirected stereotactic body radiotherapy. ${ }^{51}$ Similarly, complex and long histories of oligometastatic disease, with up to maximum of four courses of radical local treatment, have been reported for prostate cancer. ${ }^{35}$ A patient case example with multiple course of metastases-directed therapy for different states of oligometastatic disease is given in the appendix p15.

\section{Implementation}

The proposed oligometastatic disease characteristics should be assessed in all patients with cancer treated with radical local treatment for oligometastatic disease, although use of the classification as a definitive decisionmaking tool remains to be established. These characteristics should enable harmonisation of reporting of patients treated for oligometastatic disease and therefore allow outcome analyses of improved quality. In the context of clinical trials, the harmonised oligometastatic disease characterisation system will contribute to a better understanding and interpretation of study results and facilitate cross-study comparisons, as well as metaanalyses and systematic reviews. Acceptance and implementation of oligometastatic disease-related clinical trials into clinical practice will be facilitated by a clear and unambiguous classification system and nomenclature.

\section{Conclusions}

In summary, we have established a system for comprehensive characterisation of oligometastatic disease, which should be assessed in all patients with oligometastatic disease treated with radical local therapy within and outside of clinical trials. We have developed an oligometastatic disease classification system on the basis of a decision tree of five binary disease characterisation factors and we have proposed a dynamic oligometastatic state model. We envisage to teste these states of oligometastatic disease in the OligoCare prospective cohort trial (NCT03818503) to assess their prognostic value and their acceptance and compliance in routine practice.

\section{Contributors}

MG, YL, and PO designed the project. MG, YL, PO, and DHS collected data and did the literature review. MG, YL, PO, and DHS analysed the data. All authors contributed to the interpretation of data and writing and approval of the manuscript.

\section{Declaration of interests}

MG reports grants from Varian and an advisory board role at AstraZeneca, outside the submitted work. YL reports personal fees from AstraZeneca and RaySearch, outside the submitted work. AD reports grants and personal fees from Varian Medical Systems and personal fees from Medical Data Works and Roche, outside the submitted work. AMCD reports grants and personal fees from BMS and personal fees from Roche, MSD, Eli Lily, Takeda, Pfizer, and Boehringer Ingelheim, outside the submitted work. AMR reports grant from Varian Medical Systems, outside the submitted work. BT reports personal fees from Amgen, Janssen, Sanofi-Genzyme, and Myovant, and grants and personal fees from Astellas, Bayer, and Ferring, outside the submitted work. DV reports grants from RaySearch Laboratories, Sun Nuclear Corporation, and Sordina IORT Technologies, outside the submitted work. PO reports grants from Ferring, Merck, and Varian, and consultancy and advisory board roles at Janssen, Bayer, and Sanofi, outside the submitted work. The other authors declare no competing interests.

\section{Acknowledgments}

The support and contribution of Piet Dirix, from Iridium Kankernetwerk and University of Antwerp, Antwerp, Belgium, is highly acknowledged. 
References

1 Hellman S, Weichselbaum RR. Oligometastases. J Clin Oncol 1995; 13: 8-10.

2 Iyengar P, Wardak Z, Gerber DE, et al. Consolidative radiotherapy for limited metastatic non-small-cell lung cancer: a phase 2 randomized clinical trial. JAMA Oncol 2018; 4: e173501.

3 Ruers T, Van Coevorden F, Punt CJA, et al. Local treatment of unresectable colorectal liver metastases: results of a randomized phase II trial. J Natl Cancer Inst 2017; 109: djx149.

4 Gomez DR, Tang C, Zhang J, et al. Local consolidative therapy vs maintenance therapy or observation for patients with oligometastatic non-small-cell lung cancer: long-term results of a multi-institutional phase II, randomized study. J Clin Oncol 2019; 37: 1558-65.

5 Palma DA, Olson R, Harrow S, et al. Stereotactic ablative radiotherapy versus standard of care palliative treatment in patients with oligometastatic cancers (SABR-COMET): a randomised, phase 2, open-label trial. Lancet 2019; 393: 2051-58.

6 Ost P, Reynders D, Decaestecker K, et al. Surveillance or metastasis-directed therapy for oligometastatic prostate cancer recurrence: a prospective, randomized, multicenter phase II trial. J Clin Oncol 2018; 36: 446-53.

7 Parker CC, James ND, Brawley CD, et al. Radiotherapy to the primary tumour for newly diagnosed, metastatic prostate cancer (STAMPEDE): a randomised controlled phase 3 trial. Lancet 2018; 392: 2353-66.

8 Pitroda SP, Weichselbaum RR. Integrated molecular and clinical staging defines the spectrum of metastatic cancer. Nat Rev Clin Oncol 2019; 16: 581-88.

9 Lussier YA, Xing HR, Salama JK, et al. MicroRNA expression characterizes oligometastasis(es). PLoS One 2011; 6: e28650.

10 Lussier YA, Khodarev NN, Regan K, et al. Oligo- and polymetastatic progression in lung metastasis(es) patients is associated with specific microRNAs. PLoS One 2012; 7: e50141.

11 Pitroda SP, Khodarev NN, Huang L, et al. Integrated molecular subtyping defines a curable oligometastatic state in colorectal liver metastasis. Nat Commun 2018; 9: 1793.

12 Dhondt B, De Bleser E, Claeys T, et al. Discovery and validation of a serum microRNA signature to characterize oligo- and polymetastatic prostate cancer: not ready for prime time. World J Urol 2018; published online Dec 21. https://doi.org/10.1007/s00345-018-2609-8.

13 deSouza NM, Tempany CM. A risk-based approach to identifying oligometastatic disease on imaging. Int J Cancer 2019; 144: 422-30.

14 Rieber J, Abbassi-Senger N, Adebahr S, et al. Influence of institutional experience and technological advances on outcome of stereotactic body radiation therapy for oligometastatic lung disease. Int J Radiat Oncol Biol Phys 2017; 98: 511-20.

15 Samim M, Molenaar IQ, Seesing MFJ, et al. The diagnostic performance of 18F-FDG PET/CT, CT and MRI in the treatment evaluation of ablation therapy for colorectal liver metastases: a systematic review and meta-analysis. Surg Oncol 2017; 26: 37-45.

16 Lecouvet FE, Oprea-Lager DE, Liu Y, et al. Use of modern imaging methods to facilitate trials of metastasis-directed therapy for oligometastatic disease in prostate cancer: a consensus recommendation from the EORTC Imaging Group. Lancet Oncol 2018; 19: e534-45.

17 deSouza NM, Liu Y, Chiti A, et al. Strategies and technical challenges for imaging oligometastatic disease: Recommendations from the European Organisation for Research and Treatment of Cancer imaging group. Eur J Cancer 2018; 91: 153-63.

18 Planchard D, Popat S, Kerr K, et al. Metastatic non-small cell lung cancer: ESMO Clinical Practice Guidelines for diagnosis, treatment and follow-up. Ann Oncol 2018; 29 (suppl_4): 192-237.

19 Dingemans AC, Hendriks LEL, Berghmans T, et al. Definition of synchronous oligo-metastatic non-small cell lung cancera consensus report. J Thorac Oncol 2019; published online Aug 6. DOI:10.1016/j.jtho.2019.07.025.

20 Ashworth A, Rodrigues G, Boldt G, Palma D. Is there an oligometastatic state in non-small cell lung cancer? A systematic review of the literature. Lung Cancer 2013; 82: 197-203.

21 Goldstraw P, Chansky K, Crowley J, et al. The IASLC lung cancer staging project: proposals for revision of the TNM stage groupings in the forthcoming (eighth) edition of the TNM classification for lung cancer. J Thorac Oncol 2016; 11: 39-51.
22 Fong Y, Fortner J, Sun RL, Brennan MF, Blumgart LH. Clinical score for predicting recurrence after hepatic resection for metastatic colorectal cancer: analysis of 1001 consecutive cases. Ann Surg 1999; 230: 309-18, discussion 318-21.

23 Fode MM, Høyer M. Survival and prognostic factors in 321 patients treated with stereotactic body radiotherapy for oligo-metastases. Radiother Oncol 2015; 114: 155-60.

24 Tanadini-Lang S, Rieber J, Filippi AR, et al. Nomogram based overall survival prediction in stereotactic body radiotherapy for oligo-metastatic lung disease. Radiother Oncol 2017; 123: 182-88.

25 Hong JC, Ayala-Peacock DN, Lee J, et al. Classification for long-term survival in oligometastatic patients treated with ablative radiotherapy: a multi-institutional pooled analysis. PLoS One 2018; 13: e0195149.

26 Moher D, Liberati A, Tetzlaff J, Altman DG. Preferred reporting items for systematic reviews and meta-analyses: the PRISMA statement. J Clin Epidemiol 2009; 62: 1006-12.

27 Hsu C-C, Sandford BA. The Delphi technique: making sense of consensus. Practical Assessment, Research \& Evaluation 12: 1-8.

28 Niibe Y, Hayakawa K. Oligometastases and oligo-recurrence: the new era of cancer therapy. Jpn J Clin Oncol 2010; 40: 107-11.

29 Palma DA, Salama JK, Lo SS, et al. The oligometastatic stateseparating truth from wishful thinking. Nat Rev Clin Oncol 2014; 11: $549-57$.

30 Reyes DK, Pienta KJ. The biology and treatment of oligometastatic cancer. Oncotarget 2015; 6: 8491-524.

31 Correa RJM, Salama JK, Milano MT, Palma DA. Stereotactic body radiotherapy for oligometastasis: opportunities for biology to guide clinical management. Cancer J 2016; 22: 247-56.

32 Patel PH, Palma D, McDonald F, Tree AC. The dandelion dilemma revisited for oligoprogression: treat the whole lawn or weed selectively? Clin Oncol ( $R$ Coll Radiol) 2019; published online June 7. DOI:10.1016/j.clon.2019.05.015.

33 Laurie SA, Banerji S, Blais N, et al. Canadian consensus: oligoprogressive, pseudoprogressive, and oligometastatic non-small-cell lung cancer. Curr Oncol 2019; 26: e81-93.

34 Milano MT, Katz AW, Okunieff P. Patterns of recurrence after curative-intent radiation for oligometastases confined to one organ. Am J Clin Oncol 2010; 33: 157-63.

35 Decaestecker K, De Meerleer G, Lambert B, et al. Repeated stereotactic body radiotherapy for oligometastatic prostate cancer recurrence. Radiat Oncol 2014; 9: 135.

36 Butte JM, Gönen M, Allen PJ, et al. Recurrence after partial hepatectomy for metastatic colorectal cancer: potentially curative role of salvage repeat resection. Ann Surg Oncol 2015; 22: 2761-71.

37 Kim JH, Rha SY, Kim C, et al. Clinicopathologic features of metachronous or synchronous gastric cancer patients with three or more primary sites. Cancer Res Treat 2010; 42: 217-24.

38 Mekenkamp LJM, Koopman M, Teerenstra S, et al. Clinicopathological features and outcome in advanced colorectal cancer patients with synchronous vs metachronous metastases. Br J Cancer 2010; 103: 159-64.

39 Tsai M-S, Su Y-H, Ho M-C, et al. Clinicopathological features and prognosis in resectable synchronous and metachronous colorectal liver metastasis. Ann Surg Oncol 2007; 14: 786-94.

40 Ashworth AB, Senan S, Palma DA, et al. An individual patient data metaanalysis of outcomes and prognostic factors after treatment of oligometastatic non-small-cell lung cancer. Clin Lung Cancer 2014; 15: 346-55.

41 Fleckenstein J, Petroff A, Schäfers H-J, Wehler T, Schöpe J, Rübe C. Long-term outcomes in radically treated synchronous $v$ s metachronous oligometastatic non-small-cell lung cancer. BMC Cancer 2016; 16: 348.

42 Neeff H, Hörth W, Makowiec F, et al. Outcome after resection of hepatic and pulmonary metastases of colorectal cancer. J Gastrointest Surg 2009; 13: 1813-20.

43 Shukuya T, Takahashi T, Naito T, et al. Continuous EGFR-TKI administration following radiotherapy for non-small cell lung cancer patients with isolated CNS failure. Lung Cancer 2011; 74: 457-61.

44 Weickhardt AJ, Scheier B, Burke JM, et al. Local ablative therapy of oligoprogressive disease prolongs disease control by tyrosine kinase inhibitors in oncogene-addicted non-small-cell lung cancer. J Thorac Oncol 2012; 7: 1807-14. 
45 Yu HA, Sima CS, Huang J, et al. Local therapy with continued EGFR tyrosine kinase inhibitor therapy as a treatment strategy in EGFR-mutant advanced lung cancers that have developed acquired resistance to EGFR tyrosine kinase inhibitors. J Thorac Oncol 2013; 8: $346-51$.

46 Lohaus F, Zöphel K, Löck S, et al. Can local ablative radiotherapy revert castration-resistant prostate cancer to an earlier stage of disease? Eur Urol 2019; 75: 548-51.

47 Schmid S, Klingbiel D, Aeppli S, et al. Patterns of progression on osimertinib in EGFR T790M positive NSCLC: a Swiss cohort study. Lung Cancer 2019; 130: 149-55.

48 Iyengar P, Kavanagh BD, Wardak Z, et al. Phase II trial of stereotactic body radiation therapy combined with erlotinib for patients with limited but progressive metastatic non-small-cell lung cancer. J Clin Oncol 2014; 32: 3824-30.
49 Heitmann J, Guckenberger M. Perspectives on oligometastasis: challenges and opportunities. J Thorac Dis 2018; 10: 113-17.

50 Scher HI, Heller G. Clinical states in prostate cancer: toward a dynamic model of disease progression. Urology 2000; 55: 323-27.

51 Klement RJ, Hoerner-Rieber J, Adebahr S, et al. Stereotactic body radiotherapy (SBRT) for multiple pulmonary oligometastases: analysis of number and timing of repeat SBRT as impact factors on treatment safety and efficacy. Radiother Oncol 2018; 127: 246-52.

(C) 2020 Elsevier Ltd. All rights reserved. 